\title{
Rage furieuse de l'enfant transmise par un chat errant : à propos d'un cas et revue de la littérature
}

\section{Furious rabies in the child transmitted by a stray cat : about one case and literature review}

Savadogo $\mathrm{M}^{1}$, Boushab $\mathrm{MB}^{2}$, Fall-Malick F Z ${ }^{3}$, Sondo K A ${ }^{1}$, Kyélèm $\mathrm{N}^{1}$

1- Service des Maladies Infectieuses au CHU- Yalgado Ouédraogo, Burkina Faso ;

2- Service de Médecine Interne/Maladies Infectieuses, Centre Hospitalier Kiffa, Mauritanie ;

3- Institut National d'Hépato-virologie, Faculté de Médecine de Nouakchott, Mauritanie ;

Auteur correspondant : Savadogo Mamoudou Maître Assistant au Service des Maladies infectieuses du CHU Yalgado Ouédraogo ; savadoma@gmail.com,

\section{Résumé}

La rage est une zoonose grave qui sévit dans la plupart des pays en développement. Elle est transmise généralement par le chien enragé mais exceptionnellement le chat peut être source de contamination de la maladie. Nous rapportons un cas de rage furieuse transmise par un chat errant, survenu chez un enfant. Le tableau clinique s'est installé 45 jours après une morsure et griffure par un chat errant alors que l'enfant était parti tôt le matin pour ses besoins derrière une concession abandonnée. La symptomatologie se résumait à une agitation psychomotrice, des hallucinations, un délire, une photophobie, une hydrophobie, une hyperesthésie cutanée, une détresse respiratoire et une déshydratation modérée, le tout évoluant dans un contexte fébrile. Sous traitement symptomatique, il décéda 48 heures après son admission.

Il ressort la nécessité de sensibiliser les populations et le personnel de santé sur le risque rabique après une exposition à une morsure ou à une griffure par un chat.

Mots clés : rage chat-enfant

\section{Summary}

Rabies is a serious zoonosis in most developing countries. It is transmitted mainly by the dog but exceptionally the cat can be a source of contamination of the disease. We report a case of furious rabies transmitted by a stray cat, which occurred in a child. The clinical picture occurred 45 days after being bitten and scratched by a stray cat while the child had left early in the morning to relieve himself behind an abandoned concession. At his admission, the child presented clinically speaking, psychomotor agitation, hallucinations, delirium, photophobia, hydrophobia, cutaneous hyperaesthesia, respiratory distress and moderate dehydration, all evolving in a febrile context. Under symptomatic treatment, he died 48 hours after admission into the department.
Hence the need to sensitize people and health workers on rabies risk after exposure to a bite or scratches by a cat.

Key words : cat, child, rabies.

\section{Introduction}

La rage est une zoonose due à un Rhabdovirus $\mathrm{du}$ genre Lyssavirus, transmise accidentellement à l'homme par morsure, griffure, ou léchage sur peau ou sur muqueuse excoriée. Elle est responsable d'une encéphalite, toujours mortelle, lorsqu'elle est déclarée [1-3]. Malgré l'existence d'un vaccin efficace disponible depuis 1885, cette maladie exceptionnelle en Europe, reste un problème de santé publique dans de nombreux pays africains dont le Burkina Faso [4-6]. Selon l'Organisation mondiale de la santé (OMS), elle est responsable de 55000 décès par an dans le monde [4,5,7], dont 25000 sur le continent africain $[1,2,5,8,9]$. Une morsure de chien en est responsable dans plus de $99 \%$ des cas [1,10], mais la maladie peut être transmise par d'autres mammifères tels la chauve-souris, le loup, le renard, le chat, les bovins, etc. $[1,4,5,11,12]$. Nous rapportons un cas de rage transmise par un chat, avec pour objectif de rappeler les mesures de prévention de cette zoonose virale grave.

\section{Observation}

Enfant de 7 ans, ayant un ATCD de morsure par un chat errant un mois et demi plus tôt, a été référé du Centre hospitalier universitaire Charles De Gaulle de Ouagadougou pour suspicion de rage. La morsure suivie de griffure serait survenue alors que l'enfant était parti à son réveil faire ses besoins derrière une maison abandonnée. Le chat a été ensuite tué et l'enfant amené au centre de santé du village où il aurait reçu une injection de nature inconnue. La tête de l'animal tué n'a pas été envoyée au laboratoire vétérinaire pour investigation. Quarante-cinq jours après la morsure, les parents constatèrent un changement de comportement de l'enfant associé à une agitation psychomotrice. Ils 
l'amènaient en consultation au Centre de Santé et de promotion Sociale(CSPS) où il recevait une injection de nature inconnue avant son transfert dans la capitale Ouagadougou. Les parents consulteront respectivement dans une clinique de la place, puis au CHU Charles De Gaulle, et enfin au CHU Yalgado Ouédraogo. L'examen clinique à l'admission relevait une agitation psychomotrice, un délire de la fièvre, une polypnée, une tachycardie, une cicatrice ponctiforme dans la région périorbitaire gauche. L'évolution a été marquée par une aggravation du tableau clinique au deuxième jour de son hospitalisation avec une persistance de la fièvre, une agitation psychomotrice, des hallucinations, une photophobie, une hydrophobie, une hyperesthésie cutanée et une détresse respiratoire suivie de son décès.

\section{Discussion}

La rage demeure une des plus anciennes maladies infectieuses mortelles transmises principalement par la salive de chiens infectés $[5,10]$. Elle est responsable d'une encéphalite toujours fatale [5]. La transmission par le chat est peu rapportée contrairement à la transmission par le chien ou par le renard [3, 11,12]. Une étude brésilienne rapportait, une prévalence de la rage féline de $44,4 \%$ et démontrait le rôle des chauves-souris dans la transmission de la maladie aux animaux domestiques [13]. Il importe donc d'intégrer la surveillance des chiroptères dans la stratégie de lutte contre la rage [13]. L'incubation dans notre cas était de 45 jours. Elle varie de quelques jours à plusieurs mois, voire des années, en fonction du siège de la morsure ou des griffures et de la gravité des lésions. Il est en général d'un à trois mois, et dans $90 \%$ des cas de moins de six mois. Cette longue durée d'incubation devrait être mise à profit pour entreprendre rapidement la vaccination ou la sérovaccination $[3,11]$. Les enfants sont plus exposés que les adultes au risque rabique $[3,13]$ à cause de leurs activités de distraction. On estime en moyenne, que 30 à $50 \%$ des décès dus à la rage surviennent chez des enfants de moins de 10 ans $[3,4,11]$. Les manifestations cliniques de notre malade étaient celles de la forme furieuse, qui est classiquement la plus fréquente avec agitations psychomotrices, hydrophobie et aérophobie évoluant dans un contexte fébrile avec une soif intense aggravée par l'hydrophobie $[3,9,16]$. Notre patient avait eu recours à une structure sanitaire après la morsure, mais aucune prophylaxie antirabique ne lui avait été proposée par méconnaissance du risque rabique après morsure par un chat. Il persiste donc une insuffisance de compétence du personnel soignant dans la prise en charge de la rage. D'où l'importance de la formation continue, de la sensibilisation des populations et de la vaccination des animaux de compagnie dans la lutte contre cette zoonose [3,17-19].

\section{Conclusion}

Bien que le chien soit de loin le vecteur principal de la rage humaine, le chat se révèle être un vecteur non négligeable dans la chaîne de transmission de cette maladie. Il importe donc de cibler cet animal de compagnie lors des campagnes de vaccinations contre la rage. Pour la lutte contre cette zoonose il serait nécessaire de créer des centres antirabiques décentralisés, de former le personnel médical et paramédical. Tout comme l'organisation des campagnes d'abattage des chiens errant, il importe de mener des campagnes similaires pour lutter contre les chats errants. Ce cas nous interpelle sur la nécessité de sensibiliser la population sur le risque rabique après une morsure ou griffure par un chat errant.

\section{Conflit d'intérêt : Aucun}

\section{Références}

1 Dodetn B, Aguemon AR, Baba BA, et al. Lutte contre la rage en Afrique : du constat a' l'action. Bull Soc Pathol Exot 2010 ; 103 : 519.

2 Davlin SL, Vonville HM. Canine rabies vaccination and domestic dog population characteristics in the developing world: A systematic review. Vaccine 2012 ; 30 : 3492502.

3 H. Peigue-Lafeuille, H. Bourhy, D. Abiteboul et al . La rage humaine en France en 2004 : état des lieux et prise en charge. Méd et mal infect 34 (2004) 551-560

4 Susilawathi NM, Darwinata AE, Dwija et al. Epidemiological and clinical features of human rabies cases in Bali 2008-2010.BMC Infect Dis $2012 ; 12: 81$.

5 Dacheux L, Delmas O, Bourhy H. Human Rabies Encephalitis Prevention and Treatment: Progress Since Pasteur's Discovery. Infect Disord Drug Targets. 2011;11(3):251-99

6 Sondo KA, Okengo YC, Diop SA, et al. Rabies in Children: Report of 24 Cases at the Yalgago Ouedraogo University Hospital Center of Ouagadougou in Burkina Faso. J Trop Dis, 2015; 3: 168.

7 Dacheux L, Bourhy H. Le diagnostic de la rage. Revue francophone des laboratoires $2011 ; 430: 33$. 
8 Yin $\mathrm{CP}$, Zhou $\mathrm{H}, \mathrm{Wu} \mathrm{H}$, et al. Analysis on factors related to rabies epidemic in China from 2007-2011. Virol Sin 2012 ; 2 : 132-43. 9 Dao S, Abdillahi AM, Bougoudogo F, Toure $\mathrm{K}$, Simbe C. Aspects épidémiologiques de la rage humaine et animale en milieu urbain à Bamako, Mali. Bull Soc Pathol Exot 2006 ; 99 : 183-6.

10 Weyer J, Szmyd-Potapczuk AV, Blumberg $\mathrm{LH}$ et al. Epidemiology of human rabies in South Africa, 1983-2007. Virus Res. 2011; 155(1):283-90.

11 Dey AC, Shahidullah M, Hossain MA, Mannan MA, Mitra U. Human Rabies among the Paediatric Population in Bangladesh. Mymensingh Med J. 2011; 20(2):245-51

12 Dimaano EM, Scholand SJ, Alera MT, Belandres DB. Clinical and epidemiological features of human rabies cases in the Philippines: a review from 1987 to 2006 . Int J Infect Dis. 2011;15(7):e495-9.

13 Castilho JG, de Souza DN, Oliveira RN, et al. The Epidemiological Importance of Bats in the Transmission of Rabies to Dogs and Cats in the State of São Paulo, Brazil, Between 2005 and 2014. Zoonoses Public Health. 2017 Sep;64(6):423-430.

thol Exot. 2010;103(1):51-9.
14 Savadogo M, Boushab MB, Sondo K A, Dabilgou A, Kaboré J. La rage infantile à porte d'entrée céphalique (À propos de deux cas). Revue Malienne d'Infectiologie et de Microbiologie 2016 ; (7) : 60-62

15 Diop SA, Manga NM, Dia NM et al. The point on human rabies in Senegal from 1986 to 2005. Med Mal Infect. 2007 ; 37(12):78791.

16 Savadogo M., Boushab M.B. La rage chez l'enfant : un risque encore méconnu des populations exposées. Médecine et Santé Tropicales, 2015 ; 25 : 222-224

17 Sow PS, Diop BM, Faye Ndao MA, Badiane S, Coll/Seck AM. Human rabies in Senegal: epidemiological and clinical aspects. Dakar Med. 1991;36(2):105-11

16 Dodet B; Africa Rabies Bureau (AfroREB). The fight against rabies in Africa: From recognition to action. Vaccine. 2009 Aug 13; 27(37):5027-32.

19 Dodet B; le Bureau d'experts de la rage du continent africain (AfroREB), Adjogoua EV et al. The fight against rabies in Africa: from recognition to action. Bull Soc $\mathrm{Pa}$ 